\title{
REACTION RATE THEORY-BASED MATHEMATICAL APPROXIMATION FOR THE AMOUNT OF TIME IT TAKES FOR CEllular Respiration TO OCCUR
}

\author{
A PREPRINT \\ Archit Chaturvedi \\ Canyon Crest Academy \\ San Diego, California, 92130 \\ archie.4ved@gmail.com
}

\begin{abstract}
The venerable process of cellular respiration is essential for cells to produce energy from glucose molecules, in order to carry out cellular work. The process is responsible for producing molecules of ATP, a molecule which is thermodynamically coupled with other biochemical and biophysical processes in order to provide energy for such processes to occur. While the process of cellular respiration is essential to biology, one cycle of the process occurs only in a matter of milliseconds, and so, it would be impractical to measure the time it takes for the process to occur through conventional means. Therefore, using concepts from reaction rate theory, particularly Marcus Theory of electron transfer, Michaelis-Menten kinetics for enzymatic catalysis, and the hard-sphere model of collision theory, I formulate and propose a mathematical approximation for the amount of time it takes for cellular respiration to occur. Through this heuristic approach, quantitatively knowing the amount of time it takes for one cycle of cellular respiration to occur could potentially have future applications in biological research.
\end{abstract}

Keywords Reaction Rate Theory $\cdot$ Collision Theory $\cdot$ Marcus Theory $\cdot$ Michaelis-Menten Kinetics $\cdot$ Cellular Respiration · Theoretical Biochemistry · Theoretical Biophysics

\section{Introduction}

\subsection{Cellular Respiration}

Cellular respiration is an essential process in cells and living organisms. It allows cells to produce energy, in order to perform the required cellular work, including transport processes, cell division, and other metabolic processes. Cellular respiration consists of four steps, discussed in [13]. The first step of cellular respiration is known as glycolysis, where one molecule of glucose is broken down into two molecules of pyruvate, through a series of chemical reactions, each catalyzed by a distinct enzyme. The next step is known as pyruvate oxidation, where the two molecules of pyruvate from glycolysis are converted to molecules of Acetyl-CoA. These molecules of Acetyl-CoA are then used in the citric acid cycle, the next step of cellular respiration. The citric acid cycle produces molecules of NADH, a molecule essential for the production of ATP. Finally, oxidative phosphorylation uses the NADH molecules produced in the citric acid cycle to produce molecules of ATP, the main source of energy in living organisms. Oxidative phosphorylation involves an electron transport chain (ETC), where a series of reduction-oxidation (redox) reactions occur. Embedded in the ETC are four protein complexes, which serve as enzymes to catalyze the electron transfer reactions in the overall step. More details regarding the specific reactions of cellular respiration are discussed throughout the formulation of my mathematical approximation. 


\subsection{Basic Reaction Kinetics}

Reaction kinetics are a branch of physical chemistry, which allow to understand the rates of chemical reactions. The rate of reaction can be represented by a simple rate law. From [17], a rate law is a mathematical expression expressing the rate of a chemical reaction based on the concentration of reactant molecules, and is denoted in the format:

$$
r=k c_{1}^{n_{1}} c_{2}^{n_{2}} \ldots=k \prod_{i=\text { reactants }} c_{i}^{n_{i}}
$$

In equation (1) above, $k$ denotes the rate constant of the reaction, $c_{i}$ denotes the concentration of reactant $i$, and $n_{i}$ denotes the order of reactant $i$. The reaction rate of a chemical reaction can be defined as the speed at which the chemical reaction occurs. For a chemical reaction:

$$
a A+b B \rightarrow c C+d D,
$$

the reaction rate can be defined mathematically as the derivative of the concentration of a reaction's reactants and products with respect to time:

$$
r=-\frac{1}{a} \frac{d[A]}{d t}=-\frac{1}{b} \frac{d[B]}{d t}=\frac{1}{c} \frac{d[C]}{d t}=\frac{1}{d} \frac{d[D]}{d t}
$$

Both the formulations of a reaction rate shown in equations (2) and (3) are quintessential aspects for the derivation of the approximation.

\subsection{Marcus Theory of Electron Transfer}

Marcus Theory, derived by Rudolph A. Marcus in [5] and discussed for biophysical purposes in [17], serves to describe the rate of electron transfer between two chemical species, in this case $D$ and $A$. It describes the transition between two electronic states:

$$
D+A \rightarrow D^{+}+A^{-}
$$

The formulation for Marcus Theory is derived directly from the Transition State Theory expression:

$$
k_{e t}=\frac{\omega_{N}}{2 \pi} \kappa_{e t} e^{-\Delta G^{\ddagger} / k_{B} T}
$$

Here, $\omega_{N}$ is the effective frequency of nuclear motion, $\kappa_{e t}$ is the probability of transitioning between two states $D A$ and $D^{+} A^{-}$, and $\Delta G^{\ddagger}$ represents the difference in the energy of the transition state and that of the reactants. The overall derivation of Marcus Theory would be too long to include in the introduction section of the paper, so I simply state the final equation for Marcus Theory:

$$
k_{e t}=\frac{\omega_{N}}{2 \pi} \kappa_{e t} \exp \left(-\frac{\left(\Delta G+E_{R}\right)^{2}}{4 E_{R} k_{B} T}\right)
$$

In the above equation, $\omega_{N}$ still denotes the effective frequency of nuclear motion, $\kappa_{e t}$ denotes the probability of transitioning between the two electronic states mentioned above, $\Delta G$ denotes the change in free energy during the reaction, and $E_{R}$ denotes the reorganization energy of the final state. It should be stated here that $k_{e t}$ denotes the rate constant of the reduction-oxidation reaction, not the reaction rate. Further manipulations to the mathematical formulation of Marcus Theory are stated during the derivation of my approximation.

\subsection{Enzymes and Michaelis-Menten Kinetics}

Enzymes are an essential aspect of biochemical and biophysical processes, including cellular respiration. Enzymes serve to increase the reaction rate of a chemical reaction through lowering the activation energy of the reaction, which therefore results in a lower $E_{A}$ value for the reaction. Michaelis-Menten kinetics provide the standard, and the most widely used, formulation for the reaction rate of enzymatic reactions. The Michaelis-Menten equation is described in great detail in [9], [17], and [18]. Consider the chemical reaction between enzyme (denoted $E$ ) and substrate (denoted as $S$ ) below:

$$
E+S \rightleftharpoons E S \rightarrow E+P
$$

In the above reaction, let $k_{1}$ denote the rate constant of the initial reaction between enzyme and substrate, let $k_{-1}$ denote the rate constant of the initial reaction in reverse, and let $k_{2}$ denote the rate constant of the second reaction, where the product is produced from the enzyme-substrate complex. The rate of the enzymatic reaction is given by the rate of product formation and depletion of reactant:

$$
r=\frac{d c_{P}}{d t}=-\frac{d c_{S}}{d t}
$$


Therefore, the rate is given by the rate law with respect to the concentration of enzyme-substrate complex:

$$
r=k_{2} c_{E S}
$$

Using this, the Michaelis-Menten equation for the reaction rate of enzymatic catalysis is:

$$
r=\frac{k_{2} c_{T} c_{S}}{c_{S}+K_{M}}
$$

In the equation, $c_{T}$ denotes the total concentration of enzyme, regardless of whether or not it is bound to substrate molecule:

$$
c_{T}=c_{E}+c_{E S}
$$

$c_{S}$ denotes the substrate concentration, and $K_{M}$ denotes the Michaelis constant:

$$
K_{M}=\frac{k_{-1}+k_{2}}{k_{1}}
$$

\subsection{Collision Theory}

Finally, while not used extensively throughout the paper, collision theory is used to denote the ATP Synthase and Complex IV activity during cellular respiration, since the concentration of ATP Synthase and Complex IV are very difficult to calculate, and are therefore essentially irrelevant. Collision theory states that based on the relative velocities and orientations of reactant molecules, only certain collisions between the reactant molecules result in the successful production of products in a chemical reaction. In this paper, I make use of the hard-sphere approximation of collision theory (from [17]), which, as the name suggests, assumes that the reactant molecules are spherical in shape. The mathematical formulation for collision theory, using reactants $A$ and $B$, is:

$$
r=n_{A} n_{B} \rho \pi d^{2} \sqrt{\frac{8 k_{B} T}{\pi \mu}} e^{-E_{A} / R T}
$$

Here, $n_{A}$ and $n_{B}$ represent the number densities of reactants A and B. $E_{A}$ denotes the activation energy of the reaction, $k_{B}$ denotes the Boltzmann constant, $\mathrm{R}$ denotes the gas constant, and $T$ denotes temperature. $d$ denotes the distance between the centers of particles of $A$ and $B$ :

$$
d=r_{A}+r_{B},
$$

where $r_{A}$ and $r_{B}$ denote the radii of particles $A$ and $B . \mu$ represents the reduced mass of particles $A$ and $B$ :

$$
\begin{gathered}
\mu=\frac{M_{A} M_{B}}{M_{A}+M_{B}}, \\
\overline{v_{r}}=\sqrt{\frac{8 k_{B} T}{\pi \mu}},
\end{gathered}
$$

denotes the average relative velocity of the particles. While the hard-sphere approximation of collision theory assumes that the reactant molecules are spherical in shape, this is not the case in many biochemical and biophysical reactions. Therefore, $\rho$, known scientifically as the steric factor, is a crucial aspect of collision theory. It is defined as the ratio between the experimental value and the predicted value for the collision frequency ( [6]):

$$
\rho=\frac{A_{\text {observed }}}{Z_{\text {calculated }}}
$$

The steric factor makes collision theory applicable for non-spherical molecules as reactants. Together, $\rho$ and $d$ serve to give the kinetic diameter of our reactants:

$$
d_{\text {eff }}^{2}=\rho d^{2}
$$

\section{Early Mathematical Manipulations}

\subsection{Determination of Reaction Time}

The first step for formulating the approximation is to determine the amount of time it takes for a chemical reaction to occur. From equation (3), it is known for a reactant A:

$$
r=-\frac{1}{a} \frac{d[A]}{d t}
$$


This can be equated to:

$$
r=-\frac{1}{a} \frac{\Delta[A]}{\Delta t}
$$

Through cross multiplication and reorganization of the variables, the amount of time it takes for the reaction to occur $(\Delta t)$ is written as:

$$
\Delta t=\frac{-\Delta[A]}{a \cdot r}
$$

In the reactions for cellular respiration, $a$ almost always remains as 1, and therefore, the amount of time it takes for a reaction of cellular respiration to occur is finally equated to be:

$$
\Delta t=\frac{-\Delta[A]}{r}
$$

It should be noted that for reactions with two or more reactants, $A$ can be any of the reactants in the chemical reaction.

\subsection{Manipulation to Marcus Theory}

Equation (6) gives the mathematical formulation of Marcus Theory. This formulation gives the rate constant of a redox reaction. However, the overall reaction rate, not the rate constant, is of interest for the approximation. Therefore, using the simple rate law denoted in equation (1), the reaction rate of a redox reaction can be expressed as:

$$
r_{e t}=[x]^{m}[y]^{\tau} \cdot k_{e t},
$$

which is equal to:

$$
r_{e t}=\frac{\omega_{N}}{2 \pi}[x]^{m}[y]^{\tau} \kappa_{e t} \exp \left(-\frac{\left(\Delta G+E_{R}\right)^{2}}{4 E_{R} k_{B} T}\right)
$$

In the above equation, $[x]$ and $[y]$ denote the concentrations of reactants $x$ and $y$, and $m$ and $\tau$ denote the reaction order with respect to reactants $x$ and $y$ respectively.

\subsection{Manipulation to Collision Theory}

While equation (13) gives the mathematical formulation for collision theory based on the number densities of the reactants, it would be more reasonable to denote the formulation with regards to the concentration of the reactants. The concentrations of the reactants can be expressed as:

$$
[A]=\frac{n_{A}}{N_{A}} \cdot 10^{3},[B]=\frac{n_{B}}{N_{A}} \cdot 10^{3},
$$

where $N_{A}$ denotes Avogrado's constant $\left(6.022 \cdot 10^{23}\right)$. It should be noted that the number densities of the reactants are multiplied by a constant $10^{3}$, in order to change the units from $\mathrm{m}^{3}$ to $\mathrm{molL}^{-1}$. Therefore, the mathematical formulation of collision theory based on the concentration of reactants becomes:

$$
\begin{aligned}
r=\left(\left(10^{3}\right)^{2}\right)([A] & \left.\cdot N_{A}\right)\left([B] \cdot N_{A}\right) \rho \pi d^{2} \sqrt{\frac{8 k_{B} T}{\pi \mu}} e^{-E_{A} / R T} \\
& =10^{6} N_{A}^{2}[A][B] \rho \pi d^{2} \sqrt{\frac{8 k_{B} T}{\pi \mu}} e^{-E_{A} / R T}
\end{aligned}
$$

\section{Glycolysis}

In this section of the paper, the amount of time it takes for each of the reactions of glycolysis to occur is determined. For non-redox chemical reactions, the Michaelis-Menten equation is used. And for redox reactions, the mathematical formulation of Marcus Theory, introduced in equation (24), is used.

\subsection{Important Reactions and Early Equations}

Glycolysis is the very first step of cellular respiration. As explored in [2] and [4], it consists of a series of chemical reactions which convert one molecule of glucose into two molecules of pyruvate. Glycolysis results in the net production of two ATP molecules, a phenomena known as substrate-level phosphorylation. In order to discuss further manipulations 
to the Michaelis-Menten equation and Marcus Theory in order to determine the time it takes for each reaction to occur, it is essential to look at some reactions individually. The first chemical reaction of glycolysis is:

$$
\text { Glucose }+A T P \rightarrow G 6 P+A D P+H^{+}
$$

The reaction above is catalyzed by the enzyme hexokinase. Since the reaction is not a redox reaction, the MichaelisMenten equation is used. As this is the first non-redox reaction in cellular respiration, I let $\alpha=1$ denote the reaction. Equation (22) gives the amount of time it takes for a reaction in cellular respiration to occur. Therefore, the amount of time it takes for the first reaction to occur is expressed as:

$$
t \approx \frac{-\Delta\left[n_{1}\right]}{\frac{\left(k_{2}\right)_{1} c_{T_{1}} c_{s_{1}}}{K_{M_{1}}+c_{s_{1}}}}
$$

Since there is a fraction in the denominator of the equation, equation (29) can be simplified to:

$$
t \approx \frac{-\Delta\left[n_{1}\right]\left(K_{M_{1}}+c_{s_{1}}\right)}{\left(k_{2}\right)_{1} c_{T_{1}} c_{s_{1}}}
$$

Here, $\Delta n_{1}$ denotes the change in concentration of a reactant in the first non-redox reaction of cellular respiration, which in this case, can be either glucose or ATP. $c_{T_{1}}$ denotes the total concentration of enzyme during the reaction, and $c_{s_{1}}$ denotes the average concentration of substrate during the duration of the reaction:

$$
c_{s_{1}}=\frac{\left[s_{1}\right]_{i}+\left[s_{1}\right]_{f}}{2}=\frac{\left([\text { Glucose }]_{i}+[\text { ATP }]_{i}\right)+\left([\text { Glucose }]_{f}+[\text { ATP }]_{f}\right)}{2}
$$

A crucial aspect of glycolysis is the decomposition of fructose-1,6-bisphosphate into dihydroxyacetone phosphate $(D H A P)$ and glyceraldehyde-3-phosphate $(G 3 P)$. After this reaction, the next series of reactions in glycolysis occur in their respective order. However, the remaining DHAP is then converted to G3P, resulting in the remainder of the reactions in glycolysis to occur for a second round. This is how glycolysis is able to produce two molecules of pyruvate. Out of these reactions, one of them is the reduction-oxidation reaction between $\mathrm{G} 3 \mathrm{P}$ and $N A D^{+}$into NADH, which is quintessential to future steps of cellular respiration. It should be noted that the reduction of $N A D^{+}$to NADH involves the transfer of two electrons, and therefore, two redox reactions take place during this transfer. Since these are the first reduction-oxidation reactions of glycolysis, Marcus Theory is used in order to determine the time for the reactions to occur:

$$
t \approx \frac{-4 \pi \Delta\left[x_{1}\right]}{\left(c_{x_{1}}\right)^{m_{1}}\left(c_{y_{1}}\right)^{\tau_{1}} \omega_{N_{1}} \kappa_{e t_{1}} \exp \left(-\frac{\left(\Delta G_{1}+E_{R_{1}}\right)^{2}}{4 E_{R_{1}} k_{B} T}\right)}
$$

Here, $c_{x_{1}}$ and $c_{y_{1}}$ denote the average concentration of reactants $x_{1}$ and $y_{1}$ :

$$
\begin{gathered}
c_{x_{1}}=\frac{\left[x_{1}\right]_{i}+\left[x_{1}\right]_{f}}{2}, \\
c_{y_{1}}=\frac{\left[y_{1}\right]_{i}+\left[y_{1}\right]_{f}}{2},
\end{gathered}
$$

and $m_{1}$ and $\tau_{1}$ denote the reaction order with respect to reactants $x_{1}$ and $y_{1}$ respectively. Since the remaining reactions of glycolysis after the production of G3P occur twice (two rounds), it is assumed that such reactions take the same amount of time in order to occur. Therefore, the sum of the amount of time these reactions take to occur is multiplied by 2 , in order to account for both rounds of the reactions.

\subsection{Total Times for Glycolysis}

Cumulatively, there are ten non-redox reactions in glycolysis, and therefore, the total time it takes for these reactions to occur can be denoted as:

$$
t_{\text {michaelis }} \approx-\left(\sum_{\alpha=1}^{5} \frac{\Delta\left[n_{\alpha}\right]\left(K_{M_{\alpha}}+c_{s_{\alpha}}\right)}{\left(k_{2}\right)_{\alpha} c_{T_{\alpha}} c_{s_{\alpha}}}+2 \sum_{\alpha=6}^{10} \frac{\Delta\left[n_{\alpha}\right]\left(K_{M_{\alpha}}+c_{s_{\alpha}}\right)}{\left(k_{2}\right)_{\alpha} c_{T_{\alpha}} c_{s_{\alpha}}}\right)
$$

For practical purposes, I include a table with the values of $\alpha$ for the equation above, as well as the corresponding chemical reactions for these values. The table is shown as Table 1.

It should be noted that $P G$ stands for phosphoglycerate. Furthermore, it should also be noted that the substrate molecule in the numerator of equation (35) can be any of the reactants present in their respective chemical reactions in Table 1.

For now, the redox reaction in glycolysis is ignored, in which $N A D^{+}$is reduced to $N A D H$, since it in itself is irrelevant to the overall mathematical approximation. The reaction will be taken into account once pyruvate oxidation is discussed. 
Table 1: Glycolysis Reactions for Values of $\alpha$.

\begin{tabular}{c|c|c}
$\begin{array}{c}\text { Value of } \alpha \\
\alpha\end{array}$ & Chemical Reaction & Enzyme Involved \\
Reaction & Hexome \\
\hline 1 & Glucose $+A T P \rightarrow G 6 P+A D P+H^{+}$ & Phose \\
2 & $G 6 P \rightarrow F 6 P$ & Phospoglucose Isomerase \\
3 & $F 6 P+A T P \rightarrow F 1,6 B P+A D P+H^{+}$ & Phosphofructokinase \\
4 & $F 1,6 B P \rightarrow D H A P+G 3 P$ & Aldolase \\
5 & $D H A P \rightarrow G 3 P$ & Triose Phosphate Isomerase \\
6 & $G 3 P+P \rightarrow \rightarrow 3 B P G$ & G3P Dehydrogenase \\
7 & $1,3 B P G+A D P \rightarrow 3-P G+A T P$ & Phosphoglycerate Kinase \\
8 & $3-P G \rightarrow 2-P G$ & Phosphoglycerate Mutase \\
9 & $2-P G \rightarrow P E P+H_{2} O$ & Enolase \\
10 & $P E P+A D P+H^{+} \rightarrow$ pyruvate $+A T P$ & Pyruvate Kinase
\end{tabular}

\section{Pyruvate Oxidation}

Pyruvate oxidation is a relatively short aspect of cellular respiration compared to the other steps of cellular respiration. All the steps of pyruvate oxidation are catalyzed by different components of an enzyme known as pyruvate dehydrogenase. Here, I simply discuss the reactions which make up pyruvate oxidation (from [2]), and also manipulate previous mathematical formulations based on the new reactions.

\subsection{Pyruvate to Acetyl CoA: Overview of Reactions}

The oxidation of pyruvate to Acetyl-CoA consists of several chemical reactions. Overall, the steps of pyruvate oxidation can be divided into three parts: decarboxylation, oxidation, formation of Acetyl-CoA. Below, the steps of pyruvate oxidation are stated:

1. Pyruvate combines with a compound known as TPP, and forms hydroxyethyl-TPP.

2. The hydroxyethyl group attached to TPP is oxidized to form an acetyl group, and is simultaneously transferred to lipoamide. Lipoamide in this case serves as the oxidizing agent for the reaction.

3. The acetyl group from acetyllipoamide is transferred to Coenzyme-A in order to form Acetyl-CoA.

4. Dihydrolipoamide is oxidized to lipoamide, by transferring two electrons to FAD, which are eventually transferred to $N A D^{+}$, reducing it to NADH.

Next, I count the number of non-redox and redox reactions in pyruvate oxidation, and alter the mathematical approximations accordingly. I also introduce the time approximation for redox reactions.

\subsection{Mathematical Manipulations}

Based on the listed steps of pyruvate oxidation, it can be seen that there are three non-redox reactions that occur during the process:

1. Formation of hydroxyethyl-TPP

2. Transfer of hydroxyethyl group to lipoamide

3. Transfer of acetyl group from acetyllipoamide to Coenzyme-A in order to form Acetyl-CoA

Based on this, three additional terms can be added to equation (35), thus giving:

$$
t_{\text {michaelis }} \approx-\left(\sum_{\alpha=1}^{5} \frac{\Delta\left[n_{\alpha}\right]\left(K_{M_{\alpha}}+c_{s_{\alpha}}\right)}{\left(k_{2}\right)_{\alpha} c_{T_{\alpha}} c_{s_{\alpha}}}+2 \sum_{\alpha=6}^{13} \frac{\Delta\left[n_{\alpha}\right]\left(K_{M_{\alpha}}+c_{s_{\alpha}}\right)}{\left(k_{2}\right)_{\alpha} c_{T_{\alpha}} c_{s_{\alpha}}}\right)
$$

Step 2 involves one redox reaction, and Step 4 involves four redox reactions (since 4 total electrons are transferred during the step). Therefore, there are five total redox reactions in pyruvate oxidation. From glycolysis, there is another two redox reactions that take place. This adds up to a total of seven redox reactions in the approximation so far. I now introduce the mathematical approximation for the amount of time it takes for the reactions which make use of Marcus 
Theory (redox reactions) to occur. From these seven redox reactions, and using equation (24), the approximation becomes:

$$
t_{\text {marcus }} \approx-4 \pi \sum_{\beta=1}^{7} \frac{\Delta\left[x_{\beta}\right]}{\left(c_{x_{\beta}}\right)^{m_{\beta}}\left(c_{y_{\beta}}\right)^{\tau_{\beta}} \omega_{N_{\beta}} \kappa_{e t_{\beta}} \exp \left(-\frac{\left(\Delta G_{\beta}+E_{R_{\beta}}\right)^{2}}{4 E_{R_{\beta}} k_{B} T}\right)}
$$

In the above equation, $\beta=1,2$ denotes the reduction of $N A D^{+}$to $N A D H$ in glycolysis, $\beta=3$ denotes the oxidation of the hydroxyethyl group attached to TPP in Step 2 of pyruvate oxidation, $\beta=4,5$ denotes the transfer of electrons from dihydrolipoamide to $\mathrm{FAD}$, and $\beta=6,7$ denotes the transfer of electrons from FAD to $N A D^{+}$, which reduces it to $N A D H$. It should be noted that in Step 4 of pyruvate oxidation, since two electrons are transferred from dihydrolipoamide to FAD, and then to $N A D^{+}$, the time it takes to transition from the electronic states $D A \rightarrow D^{+} A^{-}$ is multiplied by a factor of 2 , to also account for the overall transition from electronic states $D A$ to $D^{2+} A^{2-}$.

\section{Citric Acid Cycle}

\subsection{Overview and Reactions}

The citric acid cycle, also known as the TCA Cycle, is the third step of cellular respiration. It was first discovered by Hans A. Krebs in [3]. In the cycle, the Acetyl-CoA from pyruvate oxidation is converted to citrate, which then goes through a series of reactions in order to produce molecules of NADH. The citric acid cycle has six non-redox reactions, and eight redox reactions which therefore make use of Marcus Theory. Details regarding the chemical reactions are used from [2] and [16].

\subsection{Mathematical Manipulations}

Since the citric acid cycle has a total of six non-redox reactions that take place, six additional terms are added to equation (27), giving:

$$
t_{\text {michaelis }} \approx-\left(\sum_{\alpha=1}^{5} \frac{\Delta\left[n_{\alpha}\right]\left(K_{M_{\alpha}}+c_{s_{\alpha}}\right)}{\left(k_{2}\right)_{\alpha} c_{T_{\alpha}} c_{s_{\alpha}}}+2 \sum_{\alpha=6}^{19} \frac{\Delta\left[n_{\alpha}\right]\left(K_{M_{\alpha}}+c_{s_{\alpha}}\right)}{\left(k_{2}\right)_{\alpha} c_{T_{\alpha}} c_{s_{\alpha}}}\right)
$$

Table 2 below shows the chemical reactions their corresponding enzymes with regards to the values of $\alpha$ which are added in equation (38):

Table 2: Citric Acid Cycle Reactions for Values of $\alpha$.

\begin{tabular}{c|c|c} 
Value of $\alpha$ & Chemical Reaction & Enzyme \\
& Acetyl-CoA + oxaloacetate $+\mathrm{H}_{2} \mathrm{O} \rightarrow$ citrate $+\mathrm{CoA}+\mathrm{H}^{+}$ & Citrate Synthase \\
14 & Citrate $\rightarrow$ cis-aconitate $+\mathrm{H}_{2} \mathrm{O}$ & Aconitase \\
15 & cis-Aconitate $+\mathrm{H}_{2} \mathrm{O} \rightarrow$ isocitrate & Aconitase \\
16 & $\alpha$-ketoglutarate $+\mathrm{CoA} \rightarrow$ succinyl-CoA $+\mathrm{CO}_{2}$ & $\alpha$-ketoglutarate dehydrogenase \\
17 & Succinyl-CoA $+\mathrm{P}_{i}+\mathrm{ADP} \rightarrow$ succinate $+\mathrm{ATP}+\mathrm{CoA}$ & Succinyl CoA Synthetase \\
18 & Fumarate $+\mathrm{H}_{2} \mathrm{O} \rightarrow$ L-malate & Fumarase
\end{tabular}

There are eight redox reactions that take place during the citric acid cycle. Therefore, eight additional terms are added to equation (37). This now gives the total amount of time, up until oxidative phosphorylation, for the redox reactions of cellular respiration to occur as:

$$
t_{\text {marcus }} \approx-4 \pi \sum_{\beta=1}^{15} \frac{\Delta\left[x_{\beta}\right]}{\left(c_{x_{\beta}}\right)^{m_{\beta}}\left(c_{y_{\beta}}\right)^{\tau_{\beta}} \omega_{N_{\beta}} \kappa_{e t_{\beta}} \exp \left(-\frac{\left(\Delta G_{\beta}+E_{R_{\beta}}\right)^{2}}{4 E_{R_{\beta}} k_{B} T}\right)}
$$

Table 3 below provides the reactions for the additional values of $\beta$ in equation (39).

So far, the time approximations account for the reactions in glycolysis, pyruvate oxidation, and the citric acid cycle. The final step of cellular respiration is oxidative phosphorylation, which results in the overall approximation of the time it takes for cellular respiration to occur. 
Table 3: Citric Acid Cycle Reactions for Values of $\beta$.

\begin{tabular}{|c|c|c|}
\hline Value of $\beta$ & Chemical Reaction & Enzyme \\
\hline $\begin{array}{l}8,9 \\
10,11 \\
12,13 \\
14,15\end{array}$ & $\begin{array}{c}\text { Isocitrate }+\mathrm{NAD}^{+} \rightarrow \alpha \text {-ketoglutarate }+\mathrm{CO}_{2}+\mathrm{NADH} \\
N A D^{+}+\mathrm{CoA} \rightarrow \mathrm{NADH}+\mathrm{CoA}^{2-} \\
\text { Succinate }+\mathrm{FAD} \rightarrow \text { Fumarate }+\mathrm{FADH} \mathrm{H}_{2} \\
\text { L-Malate }+\mathrm{NAD}^{+} \rightarrow \text { oxaloacetate }+\mathrm{NADH}+\mathrm{H}^{+}\end{array}$ & $\begin{array}{l}\text { Isocitrate dehydrogenase } \\
\alpha \text {-ketoglutarate dehydrogenase } \\
\text { Succinate dehydrogenase } \\
\text { Malate dehydrogenase }\end{array}$ \\
\hline
\end{tabular}

\section{Oxidative Phosphorylation and Final Approximation}

\subsection{Overview}

Oxidative phosphorylation is the final step of cellular respiration, and perhaps also the most intricate step of cellular respiration. It consists of an electron transport chain (ETC) and three protein complexes, which are themselves embedded in the ETC. These protein complexes are known as the respiratory complex I, III, and IV (complex II is involved in the citric acid cycle, but not in oxidative phosphorylation). Oxidative phosphorylation consists of a series of redox reactions which take place in the ETC. The protein complexes in the ETC serve as enzymes which catalyze these electron transfer reactions. This systematic electron transfer results in the formation of a proton gradient across the mitochondrial membrane, which in turn allows ATP Synthase to produce ATP from ADP and $\mathrm{P}_{i}$. In the upcoming sections, I cover the mechanisms of each protein complex, and formulate the final time approximation.

\subsection{Note on Proton Transport}

The transport of protons in the respiratory complexes is essential for the phosphorylation of ADP to form ATP. Proton transfer is thermodynamically coupled with the electron transfer reactions in the ETC. However, while proton transfer is essential to cellular respiration, it essentially occurs simultaneously to the electron transfer in the ETC, and is therefore of no relevance to the time approximation, since the redox reactions account for the time itself.

\subsection{Complex I}

Respiratory Complex I is the first protein complex in the ETC. It is also known as NADH:ubiquinone oxidoreductase. The structure of Complex I is described in [1]. The mechanism of Complex I begins when NADH transfers two electrons to flavin mononucleotide $(F M N)$, reducing it to $\mathrm{FMNH}_{2}$. FMN then transfers the electrons through a series of iron-sulfur clusters (described in [10] and [11]), before reaching ubiquinone (Q), becoming $Q^{2-}$, and finally becoming $\mathrm{QH}_{2}$ afterwards. The overall transfer of the two electrons can be summarized below:

$$
N A D H \rightarrow F M N \rightarrow N_{3} \rightarrow N_{1} b \rightarrow N_{4} \rightarrow N_{5} \rightarrow N_{6} a \rightarrow N_{6} b \rightarrow N_{2} \rightarrow Q
$$

From above, it is seen that Complex I is marked by a series of eighteen redox reactions, two separate reactions for the two electrons transferred from one molecule to the next in the chain of reactions shown in equation (40). Since the manipulations to the time approximation for redox reactions have already been described multiple times previously, there is no need to restate the manipulations to the equation. Table 4 below denotes the molecules involved in the transfer reactions shown in equation (46) based on the values of $\beta$ :

Table 4: Complex I Reactions for Values of $\beta$.

\begin{tabular}{c|c} 
Value of $\beta$ & Transfer Reaction Molecules \\
\hline 16,17 & $N A D H \rightarrow F M N$ \\
18,19 & $F M N \rightarrow N_{3}$ \\
20,21 & $N_{3} \rightarrow N_{1} b$ \\
22,23 & $N_{1} b \rightarrow N_{4}$ \\
24,25 & $N_{4} \rightarrow N_{5}$ \\
26,27 & $N_{5} \rightarrow N_{6} a$ \\
28,29 & $N_{6} a \rightarrow N_{6} b$ \\
30,31 & $N_{6} b \rightarrow N_{2}$ \\
32,33 & $N_{2} \rightarrow Q$
\end{tabular}


As seen, there are now 33 terms in the time approximation for redox reactions. Complex I also contributes to the proton gradient required for ATP Synthase to phosphorylate ADP for ATP production. Comprehensively, a total of 4 protons are transported from the mitochondrial matrix to the cytoplasm in Complex I, a consequence of the thermodynamic coupling of electron transfer and proton transport.

\subsection{Complex III}

Respiratory Complex III, also known as Q-cytochrome c oxidoreductase, is the third protein complex in the ETC. Complex III takes electrons directly from complex I ( [12]), and continues the process of the electron transport chain. A total of four protons are transported from the mitochondrial matrix to the cytoplasm. The mechanism for complex III, described in [2], begins when two molecules of $Q H_{2}$ from complex I bind to complex III consecutively. Since the reactions for each molecule of $Q \mathrm{H}_{2}$ are essentially the same, I only discuss the reactions with regards to the first molecule of $Q H_{2}$. The first $Q H_{2}$ molecule releases two electrons. The first electron flows from the molecule to a $2 \mathrm{Fe}-2 \mathrm{~S}$ center, which is more formally termed the Rieske Center. Then, the electron flows to cytochrome $c_{1}$, from which it finally flows to a molecule of oxidized cytochrome $c$, converting the cytochrome c to its reduced form. The second electron flows from $Q H_{2}$ to two heme groups within cytochrome $b$, termed hemes $b_{L}$ and $b_{H}$ respectively. Finally, the electron flows from heme $b_{H}$ to a ubiquinone $(Q)$, reducing it to a semiquinone $Q^{-}-$. The transfer reactions for the first electron can be summarized below:

$$
\mathrm{QH}_{2} \rightarrow 2 \mathrm{Fe}-2 \mathrm{~S} \rightarrow \text { cytochrome }-c_{1} \rightarrow \text { cytochrome }-c,
$$

and the transfer reactions for the second electron can be summarized below:

$$
Q H_{2} \rightarrow b_{L} \rightarrow b_{H} \rightarrow Q
$$

As stated, the reactions for the second molecule of $Q H_{2}$ are essentially the same as that of the first molecule, the only difference being that its second electron finally binds to the reduced semiquinone from the first $Q \mathrm{H}_{2}$ molecule, reducing it $Q^{2-}$, which then combines with two protons to form $Q H_{2}$ again. Cumulatively, there are twelve redox reactions that take place in Complex III. The reactions for the second molecule of $Q \mathrm{H}_{2}$ can be approximated using the reaction for the first molecule of $\mathrm{QH}_{2}$. From above, it can be concluded that there are now 45 terms in the time-approximation for redox reactions. Table 5 below denotes the transfer reactions in Complex III for the additional values of $\beta$ :

Table 5: Complex III Reactions for Values of $\beta$.

\begin{tabular}{c|c} 
Value of $\beta$ & Transfer Reaction Molecules \\
\hline 34,40 & $Q H_{2} \rightarrow 2 F e-2 S$ \\
35,41 & $2 F e-2 S \rightarrow$ cytochrome $-c_{1}$ \\
36,42 & cytochrome $-c_{1} \rightarrow$ cytochrome $-c$ \\
37,43 & $Q H_{2} \rightarrow b_{L}$ \\
38,44 & $b_{L} \rightarrow b_{H}$ \\
39,45 & $b_{H} \rightarrow Q$
\end{tabular}

\subsection{Complex IV}

Respiratory Complex IV is the final protein complex in the ETC. Also known as cytochrome c-oxidase, the protein is responsible for the transport of four protons from the mitochondrial matrix to the cytoplasm. The mechanism of the Complex IV is described in the steps below:

1. The electrons from two molecules of reduced cytochrome $c$ go through an electron transfer pathway within the protein complex. One electron stops at the $C u_{B}$ copper ion, and the other goes to heme $a_{3}$.

2. As oxygen binds, it takes up an electron from each of the ions nearby in order to form a peroxide ion $\mathrm{O}_{2}^{2-}$

3. Two additional molecules of cytochrome $\mathrm{c}$ bind and release their electrons that travel to the active center of the complex. The addition of an electron, as well as an $\mathrm{H}^{+}$, results in the reduction of the two ion-oxygen groups to $\mathrm{Cu}_{\mathrm{B}}^{2+}-\mathrm{OH}$ and $\mathrm{Fe}^{3+}-\mathrm{OH}$.

4. Reaction with two additional $\mathrm{H}^{+}$ions allows for the release of two molecules of $\mathrm{H}_{2} \mathrm{O}$, resetting the enzyme to its initial form, which is fully oxidized.

[7] provides a comprehensive model of the redox reactions that take place in the catalytic cycle of Complex IV. Based on the model, there are eight redox reactions that take place in the complex, and therefore, eight additional terms are 
Table 6: Complex IV Reactions for Values of $\beta$

\begin{tabular}{c|c} 
Value of $\beta$ & Transfer Reaction \\
\hline 46 & $C u^{2+}+e^{-} \rightarrow C u^{+}$ \\
47 & $F e^{3+}+e^{-} \rightarrow F e^{2+}$ \\
48,49 & $F e^{2+} C u^{+} \rightarrow F e^{3+} C u^{2+}+2 e^{-}$ \\
50 & $C u^{2+}+e^{-} \rightarrow C u^{+}$ \\
51,52 & $F e^{3+} C u^{+} \rightarrow F e^{4+} C u^{2+}+2 e^{-}$ \\
53 & $F e^{4+}+e^{-} \rightarrow F e^{3+}$
\end{tabular}

added to the time approximation for redox reactions. The molecules involved in the reactions can further be found in [7]. Table 6 summarizes the reactions for the additional values of $\beta$.

There are around two reactions that take place in Complex IV, that are not redox reactions. These reactions also involve two reactants. While the Michaelis-Menten equation could be used in this case, it would be very difficult to find the concentration of enzyme involved during the reactions in the protein complex. Therefore, collision theory is used to denote the reaction rate, and hence the time approximation, for these reactions. Through this, the total time it takes for the reactions to occur is given by:

$$
t_{\text {coll }} \approx \frac{-1}{10^{6} \pi N_{A}^{2}}\left(2 \sum_{n=1}^{2} \frac{\Delta\left[A_{n}\right]}{c_{A_{n}} c_{B_{n}} \rho_{n} d_{n}^{2} \sqrt{\frac{8 k_{B} T}{\pi \mu_{n}}} e^{-\left(E_{A_{n}}-\psi_{n}\right) / R T}}\right)
$$

Here, $n=1$ denotes the reaction:

$$
\mathrm{Fe}^{2+}+\mathrm{O}_{2} \rightarrow \mathrm{Fe}^{2+} \mathrm{O}_{2}
$$

and $n=2$ denotes the reaction:

$$
\mathrm{Fe}^{3+} \mathrm{O}^{2-}+\mathrm{H}^{+} \rightarrow \mathrm{Fe}^{3+} \mathrm{OH}
$$

\subsection{ATP Synthase}

ATP Synthase is responsible for the production of the remainder of the ATP molecules that are produced during cellular respiration. For ATP Synthase activity, I make use of the simple chemical reaction:

$$
A D P+P_{i} \rightarrow A T P
$$

It would be very difficult to determine the concentration of ATP Synthase, and therefore, the mathematical formulation of collision theory is used to denote the activity of the enzyme. While the theoretical yield of ATP from one cycle of cellular respiration is 38 molecules, an estimated 32 molecules of ATP are actually produced from the process. Conventionally, ATP Synthase synthesizes about 26 molecules of ATP per glucose molecule ([14]). Therefore, the time it takes for the chemical reaction to occur (based on collision theory) is simply multiplied by a factor of 26 in order to get the total time it takes for ATP Synthase to synthesize the molecules of ATP in one cycle of cellular respiration. The total time it takes for ATP Synthase to produce 26 molecules of ATP can be approximated mathematically using collision theory to be:

$$
t_{A T P} \approx \frac{-1}{10^{6} \pi N_{A}^{2}}\left(\frac{26 \Delta[A D P]}{c_{A D P} c_{\left(P_{i}\right)} \rho_{A T P} d_{A T P}^{2} \sqrt{\frac{8 k_{B} T}{\pi \mu_{A T P}}} e^{-\left(E_{A_{A T P}}-\psi_{A T P}\right) / R T}}\right)
$$

ATP in the subscript of the terms in equation (47) denotes the intended chemical reaction in equation (46). Furthermore, $c_{A D P}$ and $c_{\left(P_{i}\right)}$ denote the average concentrations of ADP and inorganic phosphate in the region:

$$
c_{A D P}=\frac{[A D P]_{i}+[A D P]_{f}}{2}, c_{\left(P_{i}\right)}=\frac{\left[P_{i}\right]_{i}+\left[P_{i}\right]_{f}}{2}
$$

$E_{A_{A T P}}$ denotes the activation energy of the reaction, and $\rho_{A T P}$ denotes the steric factor of the reaction. A value $\psi_{A T P}$ is used to denote the lowering of the activation energy of the reaction by the enzyme. It should be noted that together, $\rho_{A T P}$ and $d_{A T P}^{2}$ serve to give the kinetic diameter of ATP:

$$
d_{e f f}^{A T P}=\rho_{A T P} d_{A T P}^{2}
$$




\subsection{Final Approximation}

All that is left now to get to the final approximation is to add the final equations derived for the time approximations of the chemical reactions involved in cellular respiration:

$$
t_{\text {cell }} \approx t_{\text {michaelis }}+\left(t_{\text {coll }}+t_{\text {ATP }}\right)+t_{\text {marcus }}
$$

The total time it takes for one cycle of cellular respiration to occur is therefore denoted as:

$$
\begin{aligned}
t_{\text {cell }} \approx- & \left(\sum_{\alpha=1}^{5} \frac{\Delta\left[n_{\alpha}\right]\left(K_{M_{\alpha}}+c_{s_{\alpha}}\right)}{\left(k_{2}\right)_{\alpha} c_{T_{\alpha}} c_{s_{\alpha}}}+2 \sum_{\alpha=6}^{19} \frac{\Delta\left[n_{\alpha}\right]\left(K_{M_{\alpha}}+c_{s_{\alpha}}\right)}{\left(k_{2}\right)_{\alpha} c_{T_{\alpha}} c_{s_{\alpha}}}+4 \pi \sum_{\beta=1}^{53} \frac{\Delta\left[x_{\beta}\right]}{\left(c_{x_{\beta}}\right)^{m_{\beta}}\left(c_{y_{\beta}}\right)^{\tau_{\beta}} \omega_{N_{\beta}} \kappa_{e t_{\beta}} \exp \left(-\frac{\left(\Delta G_{\beta}+E_{R_{\beta}}\right)^{2}}{4 E_{R_{\beta}} k_{B} T}\right)}\right) \\
& -\frac{2}{10^{6} \pi N_{A}^{2}}\left(\sum_{n=1}^{2} \frac{\Delta\left[A_{n}\right]}{c_{A_{n}} c_{B_{n}} \rho_{n} d_{n}^{2} \sqrt{\frac{8 k_{B} T}{\pi \mu_{n}}} e^{-\left(E_{A_{n}}-\psi_{n}\right) / R T}}+\frac{13 \Delta[A D P]}{c_{A D P} c_{\left(P_{i}\right)} \rho_{A T P} d_{A T P}^{2} \sqrt{\frac{8 k_{B} T}{\pi \mu_{A T P}}} e^{-\left(E_{A_{A T P}}-\psi_{A T P}\right) / R T}}\right)
\end{aligned}
$$

Unfortunately, the equation, which I denote as equation (final), was too big to fit in one line and label. Regardless, this is the final approximation for the amount of time it takes for cellular respiration to occur, expressed mathematically.

\section{Discussion}

From 6.7, the final approximation for the amount of time it takes for cellular respiration to occur is shown in equation (final). The final approximation serves to provide a near-exact mathematical formulation for the time it takes for the process to occur, and can be used for mathematical, theoretical, and computational purposes. Through the approximation, I have laid out a purely mathematical means to calculate the amount of time it takes for one cycle of cellular respiration to occur.

\subsection{Assumptions and Justifications}

While the approximation serves as an accurate formulation for the amount of time it takes for cellular respiration to occur, it makes use of several assumptions during the derivation. The first such assumption is that the rate of reaction is calculated at the average concentration of the reactants. Another assumption that is made is for the redox reactions involved in cellular respiration. As seen, for a reaction where the electronic state of the molecules changes by 2 ( $D A$ to $D^{2+} A^{2-}$, the time it takes for the overall reaction to occur is approximated by multiplying, by a factor of 2 , the amount of time it takes to transition from electronic states $D A$ to $D^{+} A^{-}$. Furthermore, the time it takes for proton transport to occur is not included in the approximation, since it is assumed that the proton transport occurs simultaneously with the electron transfer reactions along the ETC. It would be of question as to why I use Marcus Theory and Michaelis-Menten kinetics for the time approximations, instead of simple rate laws. The Michaelis-Menten equation is the standard mathematical formulation of enzymatic catalysis, and is therefore the best equation to use for the time approximations of non-redox reactions. Using solely the concentrations of substrate molecules and enzyme, as well as through the rate constants of the reactions, the Michaelis-Menten equation provides a simple and efficient means to calculate the reaction rate of the chemical reaction. The equation can be used for both mathematical and computational research in biology, thus making the final approximation derived in this paper more flexible with regards to biological research. Marcus Theory of electron transfer is the most well-known means to denote a rate expression for reduction-oxidation reactions. It therefore provides the most venerable, and widely used, means to calculate the reaction rate of electron transfer reactions based on the concentration of reactant molecules, reaction orders with respect to the reactants, and the other variables discussed in equation (6). Collision theory is used to denote ATP Synthase activity and the non-redox reactions in Complex IV. This is due to the fact that it would be impractical to calculate the concentration of the enzyme. Calculating the reaction rate solely based on the concentration of reactants, kinetic diameter, activation energy, and enzymatic activity would simplify the time-approximation greatly, and therefore, it is concluded that the mathematical formulation of collision theory is the best means to denote the time it takes for Complex IV and ATP Synthase activity to occur. Finally, the approximation does not make use of the rate-limiting step in any of the steps of cellular respiration. While the rate-limiting step could potentially provide a good approximation for the reaction rate of an entire step of cellular respiration, the overall process of cellular respiration occurs in a matter of milliseconds, and therefore, it is reasonable to take into account all of the reactions which make up cellular respiration. 


\subsection{Practicality: Calculations and Uses}

The process of cellular respiration occurs in a matter of milliseconds, and may vary based on the concentration of reactants. Therefore, experimentally determining the amount of time it takes for one cycle of cellular respiration to occur would be rather impractical, as there could be potential sources of error and bias in the measured time. Hence, the final time-approximation derived in this paper serves as a plausible means to estimate for how long it takes for one cycle of the process to occur. If the values of the terms used in the final approximation are known, then they can simply be added together in order to get the final time. Calculating the initial concentration and final concentration of the reactants, as well as the reaction order, may require estimation. Furthermore, calculating the steric factor for ATP Synthase activity may also require estimation, since there is no simple way to calculate it ( [17]). However, while the steric factor is difficult to calculate, equation (18) gives the kinetic diameter for the reactants of collision theory. As ATP remains a well-known molecule in biochemistry, the kinetic diameter of each reactant can be found in a number of other research papers, and through computational tools such as JMOL as well. Furthermore, source [8] gives a method to calculate the kinetic diameter of dense liquids through the use of the Lennard-Jones potential function and internal pressure results. Perhaps a similar method can be used to calculate the kinetic diameter of the reactants in cellular respiration. Many of the values for the final approximation have been calculated experimentally in the past, and a number of such values can be found in [2]. Such values can be used, and the total time it takes for cellular respiration to occur based on the final approximation, can be found and stated in a matter of one research paper. While the approximation gives a scientifically valid approximation for the amount of time it takes for one cycle of cellular respiration to occur, it could potentially take a long time, and tedious work to sum up all of the numbers and determine the total time. Therefore, I also propose an alternative approach to find the total time it takes for one cycle of the process to occur. I have stated and laid out, both accurately and heuristically, the reactions that make up cellular respiration. Kinetically modelling each of the reactions stated in the tables using computational tools, and summing up the times it takes for the reactions to occur based on the computational models, can give the total time it takes for cellular respiration to occur:

$$
t_{\text {cell }} \approx \sum t_{\text {non-redox }}+t_{A T P}+\sum t_{\text {redox }}
$$

Equation (51) simply represents the fact that the total time it takes for one cycle of cellular respiration to occur is the sum of all the reactions discussed throughout the paper, mathematically. While this technique would not make use of the final approximation derived in this paper, it would make extensive use of the reactions laid out in the tables of this paper. Several of such computational tools for this method can be found in [15]. Finally, the final approximation provides a purely mathematical means to denote how long it takes for one cycle of cellular respiration to occur. Therefore, it can be used for further research in mathematical biology and theoretical biochemistry/biophysics. Quantitatively knowing how long it takes for cellular respiration to occur can have multitudinous applications in research regarding mathematical biology, cell/molecular biology, biochemistry, biophysics, biological/biomedical engineering, synthetic biology, and a number of other fields. The mathematics used to derive the final approximation in this paper can be used to derive time-approximations for a number of other biochemical and biophysical processes. Such processes include:
1. Gluconeogenesis
2. Photosynthesis: Light Reactions and the Calvin Cycle
3. Cori Cycle
4. Pentose-Phosphate Pathway
5. Glycogen Metabolism
6. Fatty Acid Metabolism
7. Amino Acid Catabolism,

and many others not included in this list. Comprehensively, the final time-approximation derived in this paper has many applications to biological research, both quantitatively and qualitatively.

\section{About the Author}

I am currently a high school student at Canyon Crest Academy, located in San Diego, California. I am entirely self-taught in the fields of mathematics, biology, chemistry, and physics. I first became interested in biology in middle school, and studied the subject extensively. I have always been interested in applying theoretical concepts from mathematics, physics, and physical chemistry to biological phenomena. This paper is my first attempt to apply purely theoretical concepts from physical chemistry to biology, and hope that the time-approximation derived in this paper can be of use in future research in the fields of mathematical biology and biochemistry/biophysics. 


\section{References}

[1] Rouslan G Efremov, Rozbeh Baradaran, and Leonid A Sazanov. The architecture of respiratory complex i. Nature, 465(7297):441-445, 2010.

[2] Gregory J. Gatto Jr. Lubert Stryer Jeremy M. Berg, John L. Tymoczko. Biochemistry 8th Edition. W.H Freeman and Company, 2015.

[3] Hans Adolf Krebs and William Arthur Johnson. The role of citric acid in intermediate metabolism in animal tissues. FEBS letters, 117(S1):K2-K10, 1980.

[4] Xue-bing Li, Jun-dong Gu, and Qing-hua Zhou. Review of aerobic glycolysis and its key enzymes-new targets for lung cancer therapy. Thoracic cancer, 6(1):17-24, 2015.

[5] Rudolph A Marcus. Electron transfer reactions in chemistry. theory and experiment. Reviews of Modern Physics, 65(3):599, 1993.

[6] Alan D McNaught, Andrew Wilkinson, et al. Compendium of chemical terminology, volume 1669. Blackwell Science Oxford, 1997.

[7] Hartmut Michel, J Behr, A Harrenga, and Aimo Kannt. Cytochrome c oxidase: structure and spectroscopy. Annual review of biophysics and biomolecular structure, 27(1):329-356, 1998.

[8] Vahid Moeini and Mehri Deilam. Determination of molecular diameter by pvt. International Scholarly Research Notices, 2012, 2012.

[9] James Dickson Murray. MathematicalBiology I. An Introduction. Springer, 2002.

[10] Tomoko Ohnishi. Iron-sulfur clusters/semiquinones in complex i. Biochimica et Biophysica Acta (BBA)Bioenergetics, 1364(2):186-206, 1998.

[11] Tomoko Ohnishi and Eiko Nakamaru-Ogiso. Were there any "misassignments" among iron-sulfur clusters n4, $\mathrm{n} 5$ and n6b in nadh-quinone oxidoreductase (complex i)? Biochimica et Biophysica Acta (BBA)-Bioenergetics, 1777(7-8):703-710, 2008.

[12] CI Ragan and CHRISTINE Heron. The interaction between mitochondrial nadh-ubiquinone oxidoreductase and ubiquinol-cytochrome c oxidoreductase. evidence for stoicheiometric association. Biochemical Journal, 174(3):783-790, 1978.

[13] Jane B Reece, Lisa A Urry, Michael Lee Cain, Steven Alexander Wasserman, Peter V Minorsky, Robert B Jackson, et al. Campbell biology. Number s 1309. Pearson Boston, 2014.

[14] PR Rich. The molecular machinery of keilin's respiratory chain, 2003.

[15] Srujan K. Rokkam, John W. Lawson, Peter Cross, and Richard Burns. Computational tools for modeling chemical degradation in extreme environments. TFAWS Interdisciplinary Paper Session, 2016.

[16] Dirk Roosterman and Graeme Stuart Cottrell. Rethinking the citric acid cycle: Connecting pyruvate carboxylase and citrate synthase to the flow of energy and material. International Journal of Molecular Sciences, 22(2):604, 2021.

[17] Philipp OJ Scherer and Sighart F Fischer. Theoretical molecular biophysics. Springer, 2010.

[18] Ulrich Schwarz. Theoretical Biophysics. heidelberg University, 2021. 